\title{
BMJ Global Health Can we make human plague history? A call to action
}

Laurence Baril, ${ }^{1}$ Xavier Vallès (D) , ${ }^{1}$ Nils Christian Stenseth, ${ }^{2,3}$

Minoarisoa Rajerison, ${ }^{4}$ Maherisoa Ratsitorahina, ${ }^{1}$ Javier Pizarro-Cerdá, ${ }^{5}$

Christian Demeure, ${ }^{5}$ Steve Belmain, ${ }^{6}$ Holger Scholz, ${ }^{7}$ Romain Girod, ${ }^{8}$ Joseph Hinnebusch, ${ }^{9}$ Ines Vigan-Womas, ${ }^{10}$ Eric Bertherat, ${ }^{11}$ Arnaud Fontanet, ${ }^{12}$ Yazdan Yazadanpanah, ${ }^{13}$ Guia Carrara, ${ }^{13}$ Jane Deuve, ${ }^{14}$ Eric D'ortenzio, ${ }^{13,15}$ Jose Oswaldo Cabanillas Angulo, ${ }^{16}$ Paul Mead, ${ }^{17}$ Peter W Horby ${ }^{18}$

To cite: Baril L, Vallès X, Stenseth NC, et al. Can we make human plague history? A call to action. BMJ Global Health 2019;4:e01984. doi:10.1136/ bmjgh-2019-001984

Handling editor Seye Abimbola

Received 11 September 2019 Revised 18 0ctober 2019 Accepted 19 0ctober 2019
Check for updates

(C) Author(s) (or their employer(s)) 2019. Re-use permitted under CC BY-NC. No commercial re-use. See rights and permissions. Published by BMJ.

For numbered affiliations see end of article.

Correspondence to Dr Xavier Vallès; xavier_valles04@hotmail.com

\section{INTRODUCTION}

Plague is a communicable rodent-borne disease caused by Yersinia pestis, a Gramnegative bacillus member of the Enterobacteriaceae family. As a zoonosis, plague is primarily a wildlife disease hat occasionally spills over to the human population, resulting in seasonal surges in human cases and localised outbreaks. ${ }^{12}$ The predominant clinical form among humans is bubonic plague, which, if untreated, has a lethality of $60 \%-90 \%$ but is readily treatable with antibiotics, reducing the death rate to around $5 \%$ if administered shortly after the infection. ${ }^{3}{ }^{4}$ One to two percent of all bubonic cases develop into secondary pneumonic plague, which in turn may be transmitted from person to person through respiratory droplets, producing primary pneumonic plague in close contacts. Without antibiotic treatment, pneumonic plague is nearly $100 \%$ fatal, but early antibiotic treatment substantially improves survival. ${ }^{4}$ Today, $Y$. pestis is present in at least 26 countries, with more than 30 different flea vectors and over 200 mammal host species. ${ }^{1-3}$ Although human plague cases continue to be reported from Asia and the Americas, most cases currently occur in remote, rural areas of sub-Saharan Africa, mostly in Democratic Republic of Congo and Madagascar (around 300-500 per year). ${ }^{5}$ However, large-scale transmission may also occur. During the 14th century, the Black Death, caused by $Y$. pestis, is estimated to have killed $30 \%-40 \%$ of the European population. ${ }^{1}$

It is important to emphasise that human plague is mostly a poverty-related disease. Therefore, given that population density and the absolute number of people living in extreme poverty are both increasing in

\section{Summary box}

In spite of the historical capacity to disrupt human societies, plague is currently a neglected disease.

- Given the resilience to be controlled in the wild, the focus should be put to prevent human transmission of plague.

- Research priorities include new diagnostic and therapeutic tools which are urgently needed, especially to deal with the pulmonary form of plague.

- A cross-disciplinary research approach is needed, including basic, ecological, clinical and socia sciences.

sub-Saharan Africa, ${ }^{6}$ there is no likelihood of plague being eliminated as a public health threat in the foreseeable future. However, the WHO does not consider plague to be either a neglected tropical disease or a "priority pathogen' that poses a public health risk because of its epidemic potential.

In September 2017, an unprecedented urban outbreak of pneumonic plague was declared in Madagascar, striking primarily its capital Antananarivo and the major seaport of Toamasina. ${ }^{7}$ This episode once again brought international attention to plague, reminding us of the capacity for human plague to spread in urban settings and cause substantial societal and economic disruption. This should raise alarm bells that a research agenda is needed.

\section{IMPROVED UNDERSTANDING OF HOST-VECTOR- PATHOGEN INTERPLAY}

Given the extraordinary ability of $Y$. pestis to adapt and colonise different environments, host reservoirs and vectors, and to re-emerge $^{8}$ the elimination of plague among wildlife species is judged almost impossible. 


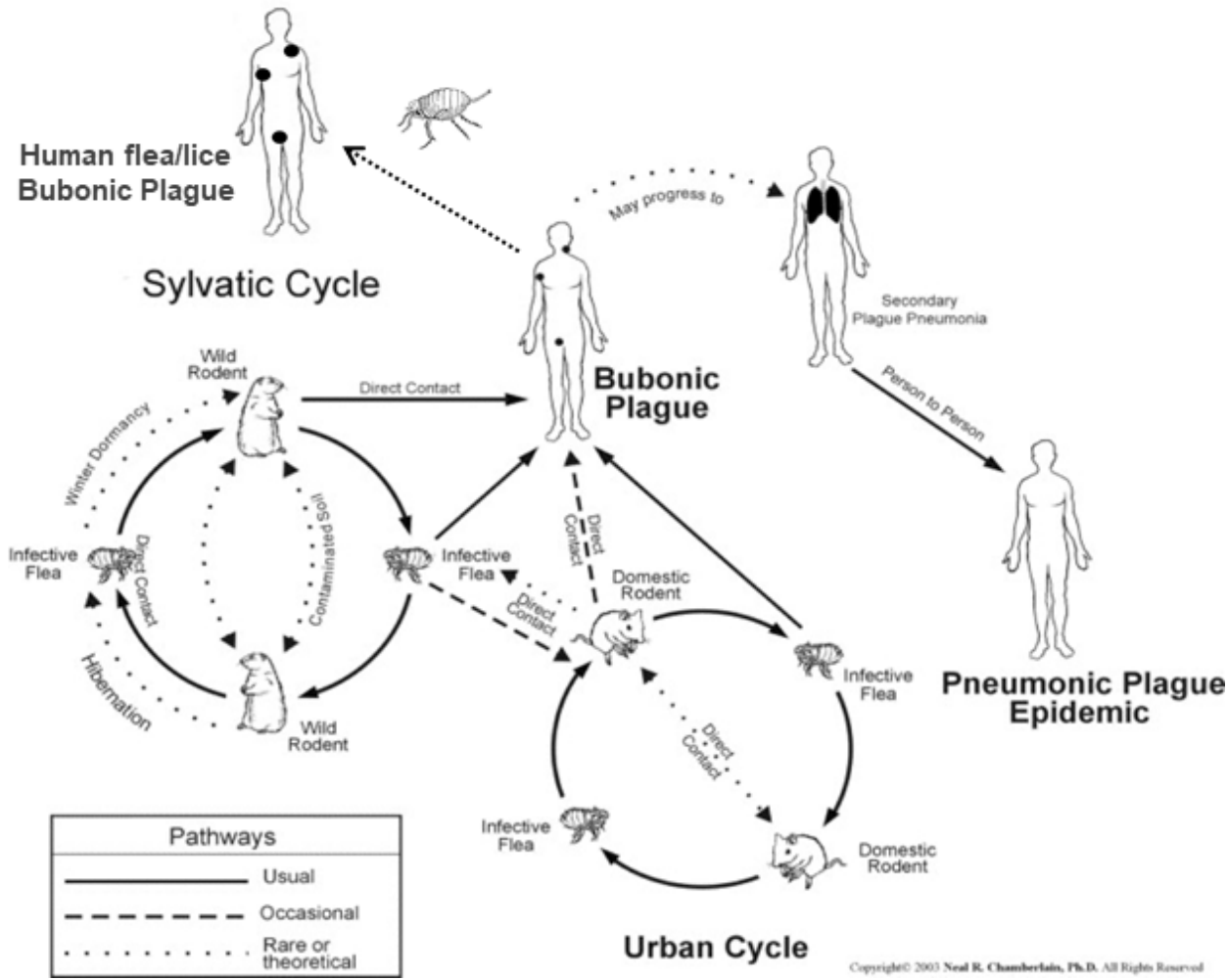

Figure 1 Epizootic/enzootic cycles of Yersinia pestis. With permission and modified from a diagram originally produced by Neal R. Chamberlain.

Therefore, the focus should be put to prevent human transmission. The risk of human plague occurrence is linked to the dynamics of enzootic (maintenance in the wild) and epizootic (episodic amplification within and between mammal species, including domestic animals) cycles, where these dynamics remain poorly understood for most wildlife systems. Understanding the biology of host-vector-pathogen interactions and proximity to humans (figure 1) is a prerequisite to address apparently simple but not well-resolved questions regarding control strategies, such as the relative efficacy of using flea control on its own or in combination with rodent control. It is not just a matter of ascertaining which are the vectors, hosts and reservoirs involved, but also understanding the internal dynamics of enzootic/epizootic cycles and the thresholds that may lead to spillover to humans. Furthermore, the existence of other unknown host and vector species, the role of human ectoparasites ${ }^{9}$ and the role of long-term persistence in fleas, postmortem tissues or in the soil still remain unclear and have to be investigated.

\section{HUMAN BEHAVIOURS AND COMMUNITY ENGAGEMENT}

Besides vector or rodent control measures, there are specific human behaviours that could be modified to avoid proliferation of domestic hosts, reduce contact with infected vectors/reservoirs and limit the potential spread of the disease. The diversity of human behaviours in different contexts may be a determinant of an epizootic cycle or for developing an adequate response. However, some aspects remain unknown, and changing deeply rooted cultural behaviours may be difficult. For instance, despite efforts for disease prevention and control, disease stigmatisation leading to a delay in seeking medical care or decisions to seek care from traditional healers can increase the risk of an outbreak. Of special concern is the connection between rural and urban areas, affecting the risk for the occurrence of major urban outbreaks. Special effort should be made towards community engagement to obtain their consent and cooperation for public health responses. For that, support from social scientists can provide valuable insights that can mitigate distrust, support cooperation and improve community communication.

\section{IMPROVEMENT OF DIAGNOSTIC TOOLS}

Early and proper diagnosis of human plague victims is critical as delayed treatment is associated with poor clinical outcomes. In endemic areas, bubonic cases can generally be diagnosed based on clinical symptoms, together with the use of rapid diagnostic tests (RDTs) to detect F1 antigen in bubo aspirates. RDTs have been used successfully as an onsite diagnostic test in Madagascar since 2002, with positive and negative predictive values of $90.6 \%$ and $86.7 \%$, respectively. ${ }^{10}$ Pneumonic cases, however, pose a significant diagnostic challenge in poorresource settings. Data from the 2017 epidemic in Madagascar suggest that there are limitations to using RDTs on sputum samples. Sputum samples can vary widely in quality, and correct processing and proper usage of the RDT are crucial parameters to get reliable results. Early 
and accurate diagnosis of pulmonary plague cases is critical for detecting and controlling outbreaks, because pneumonic plague is the most lethal form of the disease. Therefore, serious consequences can arise from both false-negative and false-positive results. Bacteriological methods (microscopy and culture) and molecular biological testing in a highly specialised laboratory are still necessary (ie, to improve selective media for cultivation or PCR antigen detection in blood).

\section{IMPROVEMENT OF CASE MANAGEMENT}

Streptomycin is highly effective and has long been considered the first choice for the treatment of most forms of plague. Nevertheless, it has a number of constraints, including adverse effects (deafness and renal toxicity), and must be administered carefully to children and pregnant women. Furthermore, since streptomycin is administered via intramuscular injection, it poses logistical problems in poor-resource settings. Indeed, treating a large number of patients with streptomycin in the context of an outbreak can be a major challenge. Since it is no longer used as a first-line treatment of tuberculosis, streptomycin is not widely available, and its distribution may cease within a few years. In addition, the lack of specific clinical and reliable onsite diagnostics for pneumonic plague could be responsible for an important number of misdiagnoses and, consequently, inadequate treatment of common respiratory infections with streptomycin. Other drugs have been successfully used for the treatment of plague and are recommended in treatment guidelines, including quinolones, gentamicin, doxycycline and chloramphenicol. ${ }^{3}$ These recommendations are based on animal studies, case reports, case series and one, small, randomised controlled trial of doxycycline versus gentamicin in 2002. ${ }^{11}$ New clinical trials are needed to demonstrate the efficacy of antibiotics with a more attractive safety, cost and availability profile than currently used regimens for both the treatment and prophylaxis of plague. To date, antibiotic resistance in $Y$. pestis has been described in three unrelated strains isolated from rats, carried by plasmids which included resistances to streptomycin and doxycycline. ${ }^{12}$ Therefore, given the ability of the Enterobacteriaceae family members to acquire any sort of antibiotic resistance and the extreme virulence of $Y$. pestis, ${ }^{13}$ it is essential to maintain a reliable antibiotic resistance monitoring system.

\section{PLAGUE VACCINES}

Further exploration of immunology, genetics and basic pathogen biology knowledge may lead to the development of more adequate prophylaxis strategies, including vaccination. Vaccines against $Y$. pestis have been developed since the early identification of the pathogen and have saved many lives in Madagascar before antibiotics became widely available, but due to the significant side effects, local or systemic reactions that were sometimes serious, the need for revaccination and the plummeting of numbers of human cases, its use was abandoned. A number of candidates are currently under development. ${ }^{14} \mathrm{rF} 1 \mathrm{~V}$ and SV1 vaccine candidates successfully passed phase II trials, and the WHO recently provided guidance for phase III evaluation in the field. ${ }^{15}$ Ideally, a human plague vaccine should be effective against pneumonic plague and able to be rapidly distributed as a countermeasure in an outbreak context.

\section{CONCLUSIONS}

Almost 125 years after the discovery of $Y$. pestis, its enduring threat to human health is a testament to the neglect of this disease. For instance, we do not have reliable means of rapidly diagnosing pulmonary plague nor a safe and effective vaccine for preventing it.The overall short-term focus should therefore be on specific research priorities that would facilitate better control of human plague: mapping plague risk, validation of treatment protocols, development of better diagnostic tools and vaccines and strengthening community-based interventions.

A cross-disciplinary approach is needed that spans preclinical research to develop improved diagnostics and vaccines; clinical research to evaluate new diagnostics, treatments and vaccines; and behavioural and social sciences to understand community perceptions and to engage communities in plague control and ecological sciences to better understand the determinants of enzootic/epizootic cycles.

The 2017, the Madagascar pulmonary plague outbreak, viewed alongside the recent large-scale Ebola outbreaks in densely populated and extremely poor areas in Africa, should be taken as a serious warning. Prior to the West Africa Ebola tragedy, there were few indicators that a massive Ebola epidemic was possible. We cannot say the same about plague; the distant and recent history are both in plain sight.

\section{Author affiliations}

${ }^{1}$ Epidemiology and Clinical Research Unit, Institut Pasteur de Madagascar, Antananarivo, Madagascar

${ }^{2}$ Centre for Ecological and Evolutionary Synthesis (CEES), Department of Biosciences, University of Oslo, Oslo, Norway

${ }^{3}$ Key Laboratory for Earth System Modelling, Department of Earth System Science, Tsinghua University, Beijing, China

${ }^{4}$ Plague Unit, Central Laboratory for Plague, Institut Pasteur de Madagascar, Antananarivo, Madagascar

${ }^{5}$ Yersinia Research Unit, National Reference Centre 'Plague \& Other Yersinioses',World Health Organization Collaborating Reference and Research Centre for Yersinia, Institut Pasteur, Paris, France

${ }^{6}$ Natural Resources Institute, University of Greenwich, Kent, UK

${ }^{7}$ Reference Laboratory for Plague, Bundeswehr Institute of Microbiology, Munich, Germany

${ }^{8}$ Medical Entomology Unit, Institut Pasteur de Madagascar, Antananarivo, Madagascar

${ }^{9}$ Rocky Mountain Laboratories, National Institute of Health, National Instittute of Allergy and Infectious Diseases, Hamilton, Ohio, USA

${ }^{10}$ Immunology of Infectious Diseases Unit, Institut Pasteur de Madagascar, Antananarivo, Madagascar

${ }^{11}$ Alert and Response Operations Programme, Communicable Disease Surveillance and Response Department, World Health Organization, Geneve, Switzerland 
${ }^{12}$ Emerging Diseases Epidemiology Unit, Conservatoire National des Arts et Métiers, Paris, France

${ }^{13}$ REACTing, Inserm, Université Paris Diderot, Paris, France

${ }^{14}$ Department of International Affairs, Institut Pasteur, Paris, France

${ }^{15}$ Service de Maladies Infectieuses et Tropicales, Hôpital Bichat - Claude-Bernard, Paris, France

${ }^{16}$ Control de Epidemia Desastres y Otras Emergencias Sanitarias, Oficina General de Epidemiologia, Ministerio de Salud de Perú, Lima, Peru

${ }^{17}$ Bacterial Diseases Branch, Division of Vector Borne Diseases, Centers for Disease Control and Prevention, Fort Collins, Colorado, USA

${ }^{18}$ Epidemic diseases Research Group Oxford (ERGO), Nutfield Department of

Medicine, University of Oxford, Oxford, UK

Acknowledgements The workshop on plague from where the contents of this manuscript arose was hosted by the Institut Pasteur (Paris, France) with financial and organisational support from the Department of International Affairs, the Unit of Emerging Disease Epidemiology Unit, and the Mathematical Modelling of Infectious Diseases Unit and REACTing-Inserm. Travel for OC was financed by the Department of International Affairs at the Institut Pasteur (Paris, France). Flights for LB and MR were financially supported by USAID (grant number AID-687-G-13-00003), Voahangy Andrianaivoarimanan and Minoarisoa Rajerison travel was supported by Northern Arizona University (through HDTRA1-11-16-BRCWMD-BAA). Attendance of Steven Belmain was supported by the African Union (grant numbe AURGII/1/006/2016). Flights and accommodation for FR and accommodations for RG, BR, VA and MR were financially supported by the Wellcome-Trust (grant number 211309/Z/18/Z) and REACTing-Inserm. Each of the other participants financially supported their travels attendance through their own institutional funding. Provided here is a full list of the other workshop participants with their institutions (by alphabetical order): Voahangy Andrianaivorimanana, Plague Unit, Institut Pasteur de Madagascar, Antananarivo, Madagascar; Fabrice Biot, Institut de Recherche Biomédicale des Armées, France; Simon Cauchemez, Infectious Diseases Mathematical Modelling Unit, Institut Pasteur, Paris, France; Rob Cohen, USAID, Washington, DC, USA; Koussay Dellagy, Department of International Affairs, Institut Pasteur, Paris, France; Nathalie Denoyes, Institut Pasteur, Paris, France; Hebert Echenique-Rivera, Yersinia Unit, Institut Pasteur, Paris, France; Florence Fouque, TDR, WHO, Lyon, France; Stephen Francesconi, Defence Threat reduction, Agency, Washington, DC, USA; Anna Funk, Emerging Diseases Epidemiology Unit, Institut Pasteur, Paris, France; Finnian Hanrahan, DG Research, European Commission, Brussels, Belgium; Mireille Harimalala, Medical Entomology Unit, Institut Pasteur de Madagascar, Antananarivo, Madagascar; Nadia Khellef, Institut Pasteur, Paris, France; Anne-Sophie Le Guern, Yersinia Unit, Institut Pasteur, Paris, France; Nadine Lemaitre, Bacteriology Department, CHU Lilee, France; Jean-Claude Manuguerra, CIBU, Institut Pasteur, Paris, France; Jodie Mac Vernon, GLOBID-R, Australia; Serge Morand, CIRAD, Bangkok, Thailand; Birgit Nikolay, Infectious Diseases Mathematical Modelling Unit, Institut Pasteur, Paris, France; Juliette Paireau, Infectious Diseases Mathematical Modelling Unit, Institut Pasteur, Paris, France; Anna Paoletti, Ministry for Higher Education and Research, Paris, France; Feno MJ Rakotoarimanana, Epidemiology and Clinical Research Unit, Institut Pasteur de Madagascar, Antananarivo, Madagascar; Beza Ramasidrazana, Plague Unit, Institut Pasteur de Madagascar, Antananarivo, Madagascar; Zely Arivelo Randriamanatany, Ministry of Public Health, Antananarivo, Madagascar; Cathy Roth, Department for International Development, London, UK; Alex Salam, Epidemic diseases Research Group 0xford (ERGO), Nuffield Department of Medicine, University of Oxford, Oxford, United Kingdom; Florent Sebbane, Institut Pasteur de Lille, France; Christophe Shako, Department of Disease Control, Ministry of Health, Democratic Republic of Congo; Sandra Telfer, Aberdeen University, UK; Quirine Ten Bosch, Epidemiology and Clinical Research Unit, Institut Pasteur de Madagascar, Antananarivo, Madagascar; Pablo Tortosa, la Reunion University, France; Kathleen Victoir, Department of International Affairs, Institut Pasteur, Paris, France; David Wagner, Northern Arizona University, USA.
Contributors All authors have provided substantial contributions to the conception or design of the work, drafting the work or revising it critically. All authors have provided final approval of the version published.

Competing interests None declared.

Patient consent for publication Not required.

Provenance and peer review Not commissioned; externally peer reviewed. Data availability statement № data are available.

Open access This is an open access article distributed in accordance with the Creative Commons Attribution Non Commercial (CC BY-NC 4.0) license, which permits others to distribute, remix, adapt, build upon this work non-commercially, and license their derivative works on different terms, provided the original work is properly cited, appropriate credit is given, any changes made indicated, and the use is non-commercial. See: http://creativecommons.org/licenses/by-nc/4.0/.

\section{ORCID iD}

Xavier Vallès http://orcid.org/0000-0002-4445-1490

\section{REFERENCES}

1 Stenseth NC, Atshabar BB, Begon M, et al. Plague: past, present, and future. PLOS Med 2008;5:e3.

2 Bramanti B, Stenseth NC, Walløe L, et al. Plague: a disease which changed the path of human civilization. Adv Exp Med Biol 2016;918:1-26.

3 Denis DT, Gage KL, Gratz N, et al. Plaque manual, epidemiology, distribution, surveillance and control, 1999. Available: http://www. who.int/csr/resources/publications/plague/whocdscsredc992a.pdf? ua $=1$

4 Pollitzer R, studies P. VIII. Plague studies. VIII. Clinical aspects. Bull World Health Organ 1953;9:59-129.

5 Bertherat E. Plague around the world, 2010-2015. Weekly Epidemiological Report WHO, 2016. Available: http://apps.who.int/ iris/bitstream/handle/10665/254296/WER9108_89-93.pdf

6 World Bank. Poverty in a rising Africa, 2016. Available: http://www. worldbank.org/en/region/afr/publication/poverty-rising-africapoverty-report

7 Randremanana R, Andrianaivoarimanana V, Nikolay B, et al. Epidemiological characteristics of an urban plague epidemic in Madagascar, August-November, 2017: an outbreak report. Lancet Infect Dis 2019;:1473-3099:30730-8.

8 Bertherat E, Bekhoucha S, Chougrani S, et al. Plague reappearance in Algeria after 50 years, 2003. Emerg Infect Dis 2007;13:1459-62.

9 Dean KR, Krauer F, Walløe L, et al. Human ectoparasites and the spread of plague in Europe during the second pandemic. Proc Natl Acad Sci U S A 2018;115:1304-9.

10 Chanteau S, Rahalison L, Ralafiarisoa L, et al. Development and testing of a rapid diagnostic test for bubonic and pneumonic plague. The Lancet 2003;361:211-6.

11 Mwengee W, Butler T, Mgema S, et al. Treatment of plague with gentamicin or doxycycline in a randomized clinical trial in Tanzania. Clin Infect Dis 2006;42:614-21.

12 Galimand M, Carniel E, Courvalin P. Resistance of Yersinia pestis to antimicrobial agents. Antimicrob Agents Chemother 2006;50:3233-6.

13 Welch TJ, Fricke WF, McDermott PF, et al. Multiple antimicrobial resistance in plague: an emerging public health risk. PLoS One 2007;2:e309.

14 Verma SK, Tuteja U. Plague vaccine development: current research and future trends. Front Immunol 2016;7:602.

15 WHO. Plague vaccines workshop April 23 2018, 2018. Available: http://www.who.int/blueprint/what/norms- standards/Plague_ vaccines_workshop-23-april-2018 